\title{
Design and Implementation of EOG Signal Based Wireless System to Control Smart Devices
}

\author{
Abdalrahman SKHETA ${ }^{1 *}$ (D), Nazmi EKREN ${ }^{2}$ (D) \\ 1*Marmara University, Electrical and Electronic Engineering Department, 34854, Istanbul, Turkey. (e-mail:eng.abdulrahman.sk@gmail.com). \\ ${ }^{2}$ Marmara University, Electrical and Electronic Engineering Department, 34854, Istanbul, Turkey. (nazmiekren@marmara.edu.tr).
}

\section{ARTICLE INFO}

Received: Sep., 15. 202

Revised: Oct., 21. 2021

Accepted: November, 01. 2021

Keywords:

EOG

ALS

Vital-Signals

Corresponding author: A. Skheta

ISSN: 2536-5010 | e-ISSN: 2536-5134

\section{ABSTRACT}

Vital signals like Electroencephalogram (EEG), Electrooculography (EOG), and Electroretinogram (ERG) have been used in many areas due to their effectiveness and accuracy. Electrodes can obtain the signals, and then they can be amplified and processed. This study helps people with reduced mobility like paralysis and Amyotrophic Lateral Sclerosis (ALS) using EOG signals. EOG signal is effective in this case because these people can use their eyes without any obstacles. EOG aid system can enable them to rely on themselves in the use of smart devices. This is one of the first studies to present a detailed analysis of the signal acquisition and processing and the results were promising with a result that scored $95 \%$ rate.

DOI: https://doi.org/10.36222/ejt.996015

\section{INTRODUCTION}

Helping people with disabilities is one of the most important and humane jobs. Supporting disabled people can help transient them from an inactive status in the community to active individuals who can perform daily activities independently and eventually contribute to society's building. Vital signals like (EEG), (EOG), and (ERG) have been used in many domains for medical treatment, for instance, the analysis of brain signals and capturing it for disease identification, and discovering muscle disorders. It is proved to be a promising solution for people with special needs, as these signals can be easily obtained using electrodes that can capture these signals, and then apply a set of techniques to amplify them and process them. The experiment on people with reduced mobility like paralysis and (ALS) using EOG signals is effective in this case because these people can use their eyes without any obstacles, which allows them to extend their physical capabilities with the help of technology. Such as giving commands to a computer through a mouse cursor without having direct physical contact with it. There have been useful case studies in this field that discussed various methodologies and applications, for instance, the article written by Manuel Merino (2010) [1], included developing an EOG signal processing algorithm method to detect the direction of eye movements. Another article written by Octavio Rivera, et al. (2013) [2], used an algorithm that captures the EOG signal and converts this vital signal into a mechanical one [3], which helped study and design a motorized wheelchair with EOG control for disabled people. Nese Ozkan, et al. (2017) [4], studied the design and acquisition of EOG based interactive communications for patients with ALS by applying the EOG signal to them. In the article written by Lawrence Y. Deng Chun Liang Hsu (2002) [5], an EOG-based Human-Computer interface system was developed. The article written by Lawrence R. Barea, et al. (2012) [6], discussed codification of captured EOG signals from eye movement was used for human-computer. In the article written by CristianCezar Postelnicu (2012) [7], a method to develop an EOGbased visual navigation interface by using HCI interface was studied. In the article written by A. Schlögl, C.Keinrath (2007) [8], Merging EEG signal with EOG signal to improve the quality of EOG signal was studied. In a thesis written by Ramis Ileri (2010) at Erciyes University, the determination of the most appropriate writing characters for educating children with dyslexia by using EOG signal was studied [9]. In a thesis written by Ahmet Coşkun (2017) at Selcuk University, sleep staging with (EOG) signals by 36 features was studied [10]. The advantage of this study is the ability to obtain the EOG vertical and horizontal signal simultaneously. The system is wireless; hence it can provide more convenience to the user. Vertical and horizontal eye movement is processed by a dedicated microprocessor device, and another customized 
processor is responsible for operating the connected smart device. In this way, the system is efficient as it can operate in a fast and smooth manner

\section{METHOD AND MATERIAL}

As all smart devices have the same control method, one case was studied, which is controlling the mouse cursors of a computer. The system consists of two parts (two electric circuits): the main circuit that the user places in his pocket and a second circuit placed in the smart device. The main circuit includes an effective EOG signal acquisition section. The most important components of this circuit are the instrument amplifier, second-order low pass filter, adder amplifier, ATMEGA-328 microprocessor, and HC-05 Bluetooth module. The analog EOG signal is converted into a digital signal by the microprocessor. The digital EOG signal is processed to identify the location and movement of the eye. Then the main circuit sends the data (eye location) to the second circuit via (HC-05) Bluetooth module. Where the second circuit receives the data and moves the mouse cursor of the device (computer). The second circuit includes an Arduino Leonardo and HC-05 Bluetooth module. Arduino Leonardo has a feature that can be used as a USB mouse or keyboard. Using the five electrodes placed on the head, the user can control the cursor that appears on the screen just by looking at the exact location where the user wants to move the cursor.

\subsection{The structure of the eye}

The eye is the main organ of the visual process. It is responsible for grasping events happening outside the body. Figure 1 shows major eye departments. The eye can be compared to a camera in general. Both have a lens on the front. It is responsible for projecting images onto sensitive areas at back. While a camera contains photosensitive photographic film, there is a layer called the retina in an eye. Images falling on the retina are taken by millions of nerves endings here, transmitted to the center of vision in the brain, and the image is perceived [11].

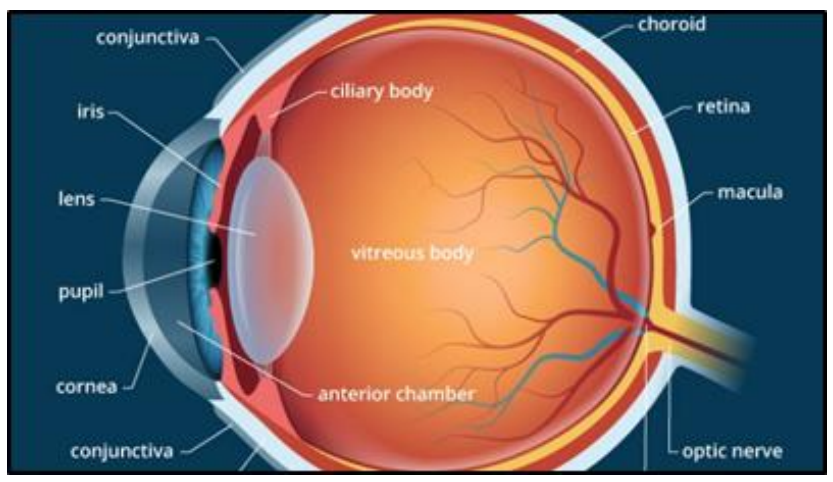

Figure 1. The Major Eye Departments [12]

\subsection{Amyotrophic lateral sclerosis (ALS)}

Diseases such as paralysis and ALS cause people to lose some basic motor skills, especially in their hands or feet. ALS, also known as motor neuron disease, is caused by the loss of motor nerve cells (neurons) in the central nervous system, spinal cord, and brainstem area. With the loss of these cells, muscle weakness and melting (atrophy) begin. On the other hand, paralysis is a clinical problem caused by brain damage caused by the cessation of flow in blood vessels feeding the brain due to a clot. The limb (s) where this brain damage occurs can no longer be controlled. Figure 2 shows the difference between an average person and a person with ALS.

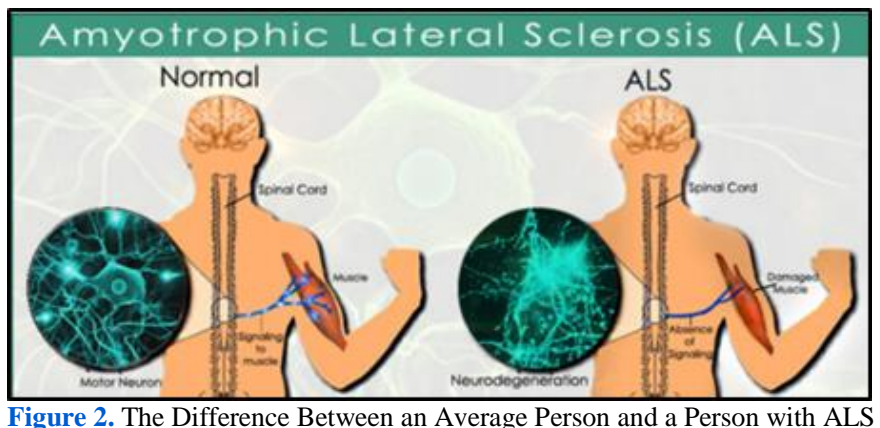
[13]

\subsection{The origin of eye vital signals}

To understand these signals, it is necessary to know eye anatomy. The eye is not a perfect sphere. It is a fused two-part unit. It consists of the anterior segment and posterior segment. The anterior segment consists of the cornea, iris, and lens. The larger posterior segment consists of the vitreous, retina, choroid, and outer white shell called the sclera. There is a permanent electrical potential difference between the anterior segment and the posterior segment. This potential arises from the retinal pigment epithelial layer. Two different vital signals can be extracted from this potential difference: ERG signal and EOG signal.

\subsubsection{ERG signal}

To measure the electrical responses of various cell types in the retina, ERG can be used. Figure 3 shows the electrical response of eye cells according to cell type. The signal is too small and normally measured in microvolts or nano-volts.

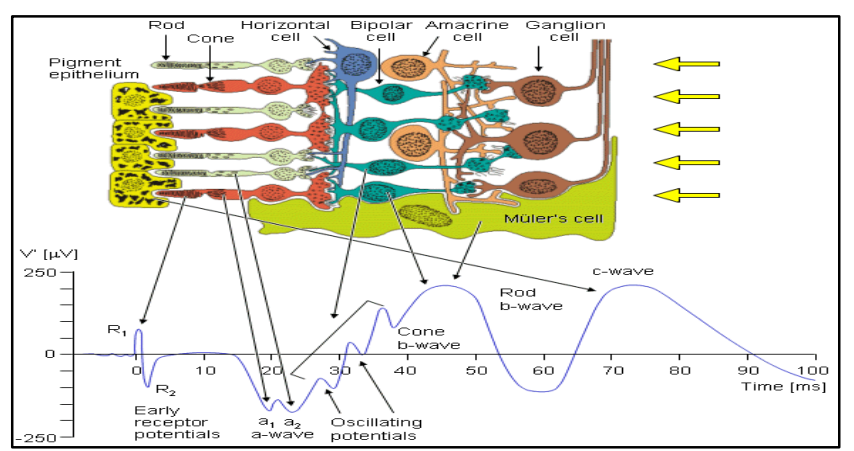

Figure 3. Review of Electrical Signals (ERG) [14]

\subsubsection{EOG signal}

The eye can be compared to an electrical dipole, where the positive pole is a cornea and the negative pole is the retina. The difference is thought to be due to the high metabolic rate in the retina. EOG is the process of recording the potential difference between the front and back of the eye, this potential difference is obtained with special electrodes that are placed appropriately around the eye [15]. Figure 4 shows EOG's electrical response as a function of time when looking left and right.

The range of the EOG signal is between 0.4 and 1.0 millivolts (without amplification). When recording a signal, it is difficult to determine the degree of eye accurately, so the achieved 
accuracy is up to \pm 2 degrees, sensitivity is between $5 \& 20$ microvolts per degree.

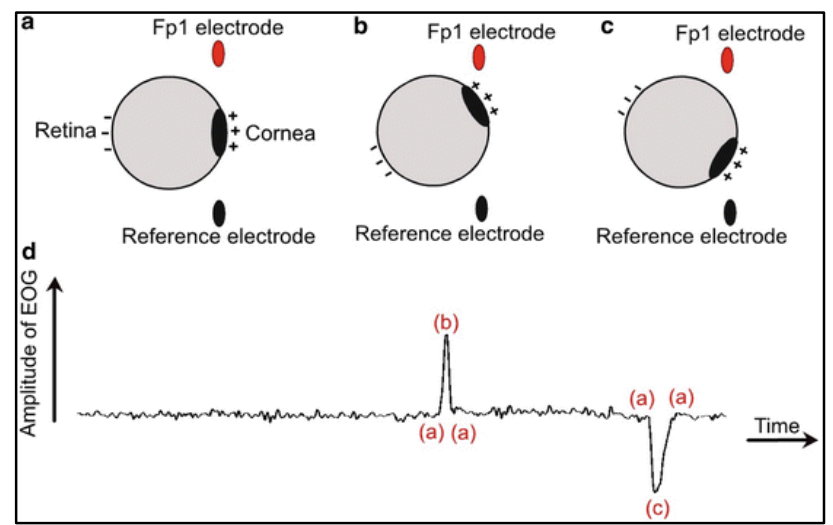

Figure 4. EOG Signals as biometrics when looking left and right [16]

The recorded EOG signal is represented as a function of time and is caused by the eye moving to a certain degree over a period of time. The difference between EOG and ERG is shown in table 1 .

TABLE II

THE DIFFERENCE BETWEEN EOG AND ERG

\begin{tabular}{lll}
\hline \multicolumn{1}{c}{ Signal } & \multicolumn{1}{c}{ ERG } & \multicolumn{1}{c}{ EOG } \\
\hline $\begin{array}{l}\text { The range of the } \\
\text { signal }\end{array}$ & $-125>>200 \mathrm{uV}$ & $0.4>>1.0 \mathrm{mV}$ \\
$\begin{array}{l}\text { Type of electrode } \\
\text { used }\end{array}$ & $\begin{array}{l}\text { Fiber electrodes } \\
\text { Uses }\end{array}$ & $\begin{array}{l}\text { Silver-silver chloride electrodes } \\
\text { Measure the } \\
\text { electrical } \\
\text { response of } \\
\text { optical eye cells }\end{array}$ \\
\end{tabular}

\subsection{Obtaining EOG signal}

EOG signal is obtained by special electrodes. Because the received signal is very low, it must be processed to become a signal that can be used. Figure 5 shows simply how the signal is obtained and amplified using an amplifier. The steps will be explained in more detail in the following paragraphs.

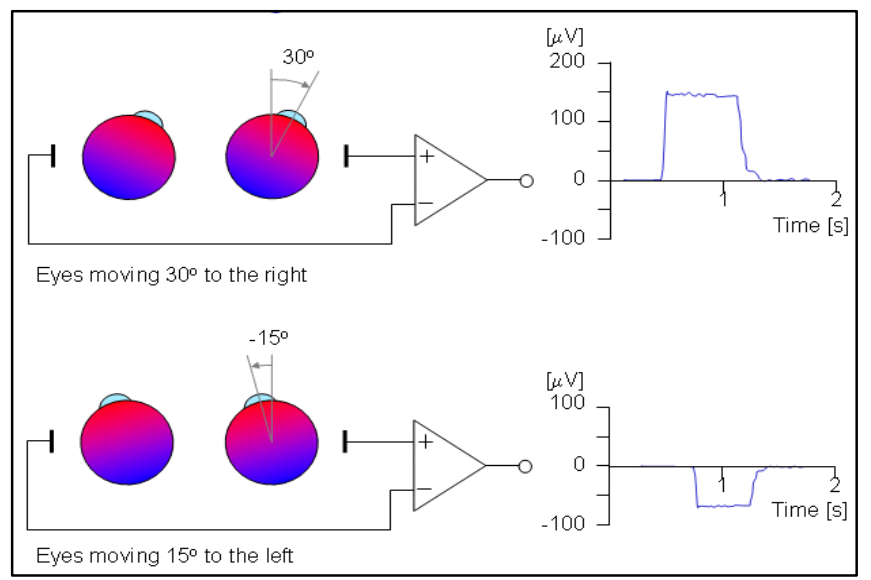

Figure 5. EOG signal Obtaining [17]

\subsection{Places where electrodes must be placed to obtain EOG}

To obtain the vertical and horizontal signals, four electrodes must be used. To reduce overlapping signal noise, a reference electrode must be placed on the forehead. Two electrodes are placed to right and left of the eye to record the horizontal signal in the eye, and two electrodes are placed below and above the eye to record the vertical signal. Figure 6 shows electrode fixation positions to determine horizontal and vertical eye movement.
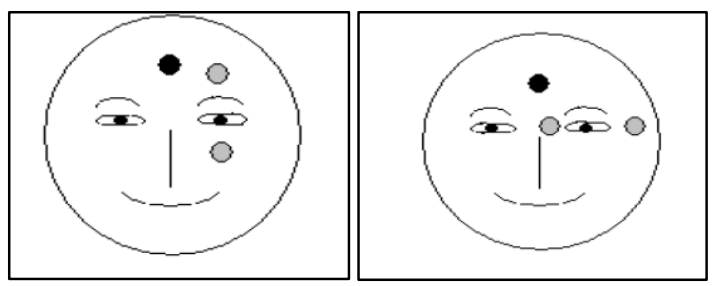

Figure 6. Electrodes placement [18]

\subsection{Diffuculties while measuring EOG and the procedures must followed}

The main difficulty is that person applying electrodes should avoid moving the muscles of the head or face as this will interfere with the signal. While using this signal to control smart devices, it is difficult to keep up the stability of these muscles during prolonged use of the signal. Filtration circuits are usually used to solve this problem. The most important steps considered to increase the accuracy of measurement:

A- Use appropriate electrodes to improve the signal.

B- Adding gel to increase conductivity of electrodes.

C- Before applying electrodes, the skin should be thoroughly cleaned.

D- Suitable insulated cables must be used to prevent electrical signals from overlapping.

\subsection{Amplifying the EOG signal}

The special operational amplifiers which is used to process vital signals from the human body is called instrument amplifiers [19]. Important signals acquired from the human body often have small amplitudes. It is challenging to obtain necessary information from small values, so the signal must be amplified and filtered. The instrument amplifier is the best combination to achieve amplification of the vital signal. It depends on the amplification of the difference between two signals in inverting and non-inverting inputs. Figure 7 shows the internal structure of the instrument amplifier.

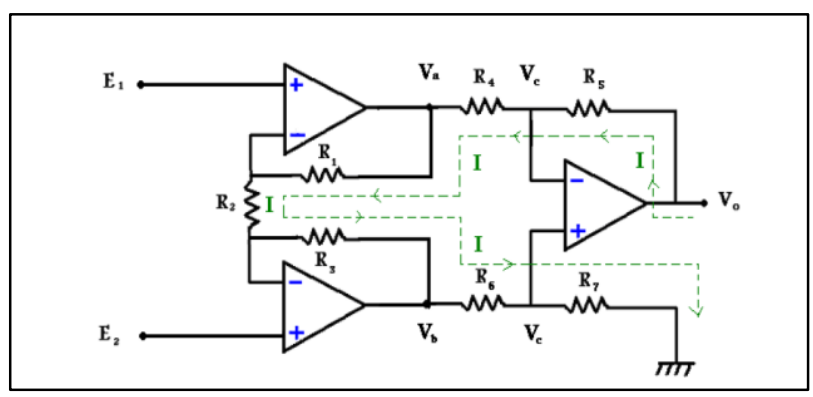

Figure 7. Internal structure of instrument amplifier [19] 
The gain of this amplifier can be controlled by changing the resistance value of R2 (External resistance) according to the following equation:

$$
\text { Gain }=1+\left(\frac{50000}{R 2}\right)
$$

To amplify signal 126 times, $400 \Omega$ resistance must be used.

\subsection{EOG signal filtering}

After amplifying the vital signal, it must be filtered from the interfering signals. Filters are electronic circuits used to prevent certain frequencies from passing through; these frequencies are noise superimposed on the frequency of the useful signal. To eliminate these frequencies, the properties of capacitors and coils were used to obtain passive filters [20]. Amplifiers are used to obtain effective filters.

\subsubsection{Effective low-pass filter}

The second-order effective low-pass filter can be used to get a better response, second-order low-pass filter is widely used as it's easy to design and operate [21]. Figure 8 shows the electric circuit of a second-order low-pass filter. Different values for $\mathrm{R} 1, \mathrm{R} 2, \mathrm{C} 1$, and $\mathrm{C} 2$ were tried until a cutoff frequency of $7 \mathrm{~Hz}$ was obtained. (When experimenting, it was observed that the best value of cut-off frequency is $7 \mathrm{~Hz}$ ). To obtain a cutoff frequency of $7 \mathrm{~Hz}$, the following values were selected;

$\mathrm{R} 3=\mathrm{R} 4=220 \mathrm{k} \boldsymbol{\Omega}$., $\mathrm{C} 1=\mathrm{C} 2=10 \mathrm{nf}$.

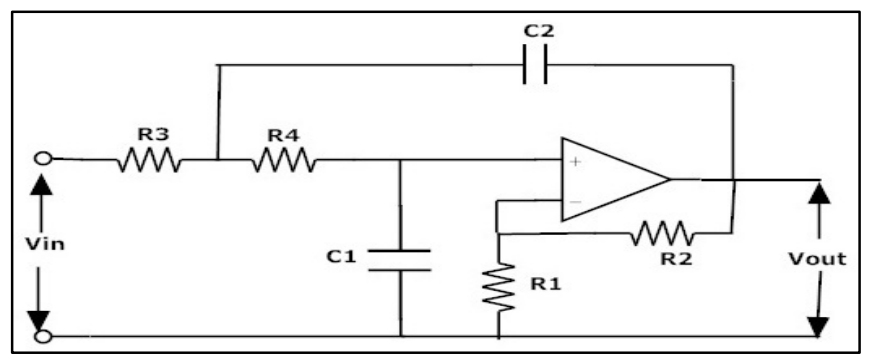

Figure 8. Effective low pass filter [22]

\subsection{Adder amplifier}

After filtering the signal, it must be converted into a digital signal, the microprocessor can be used to convert an analog signal into digital. But analog EOG signal ranges between positive and negative values. Since the microprocessor can't read negative values, another amplifier must be used to raise the negative portion of the signal and make it a positive value [23]. The best solution to increase the negative portion is using an adder amplifier. Figure 9 shows the adder amplifier circuit. This circuit is similar to an inverting operational amplifier circuit except that it has multiple inputs instead of a single input.

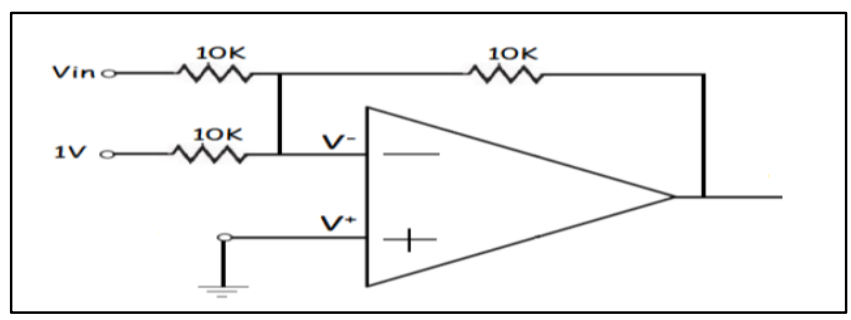

Figure 9. Adder amplifier [24]
When applying $1 \mathrm{~V}$ to $\mathrm{V}^{-}$in-circuit showed in Figure 9, and applying an analog signal to $\mathrm{V}_{\text {in }}( \pm 2)$. The output signal must be $V_{\text {in }}+1$. Figure 10 shows $V_{\text {in }}$ signal. Figure 11 shows the output signal. Likewise, the negative part of EOG signal can be amplified. The EOG signal is applied to $\mathrm{V}_{\text {in }}$. On other input, to control value it must be added to EOG signal to increase it from negative part, a voltage divider must be used.

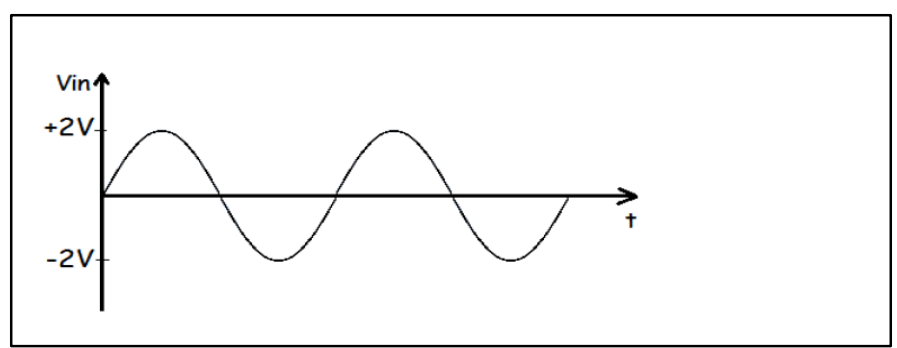

Figure 10. Adder amplifier input [24]

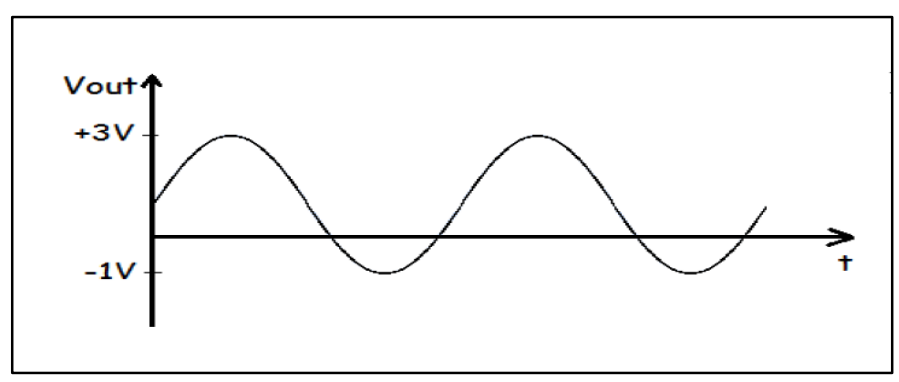

Figure 11. Adder amplifier output [24]

\subsection{Circuit Installation and components}

To amplify the vital signal, INA118 instrument amplifier was used. Then the signal is filtered with an effective low-pass filter. Next, the signal value is raised via an adder amplifier. A TL082 IC is used to filter and increase signal, where TL082 IC contains two amplifiers inside it. After obtaining the signal and filtering it, it was converted into a digital signal via ATMEGA-328p microprocessor [25]. The task of the processor in this circuit is to convert an analog signal into a digital signal and process signal to determine horizontal and vertical eye position, then send data to HC-05 Bluetooth module via UART serial port [26]. The Bluetooth module converts data into wireless signals and sends it to the second circuit. The second circuit contains a Bluetooth module and Arduino Leonardo. Bluetooth module decodes received wireless signal to obtain data, then sends data to Arduino Leonardo via serial port UART, Arduino moves mouse cursor according to received data. The Arduino Leonardo is used for this task because it has a USB connection feature and it can be programmed easily to be used as a mouse. The mission of the Arduino Leonardo is limited to converting data format from UART to USB. Two batteries were used to power the circuit (negative and positive voltages are required for the operational amplifier). It is preferable to use a battery in those circuits to reduce noise resulting from alternating current.

\subsubsection{Block diagram}

The block diagram shows the stages of signal acquisition, amplification, filtering, processing, and converting it to digital, then sending it via Bluetooth to the second circuit. The second circuit moves the mouse cursor according to data coming from the main circuit, where the circuit is fed with continuous \pm 12 volts through two batteries. Figure 12 shows the block diagram. 


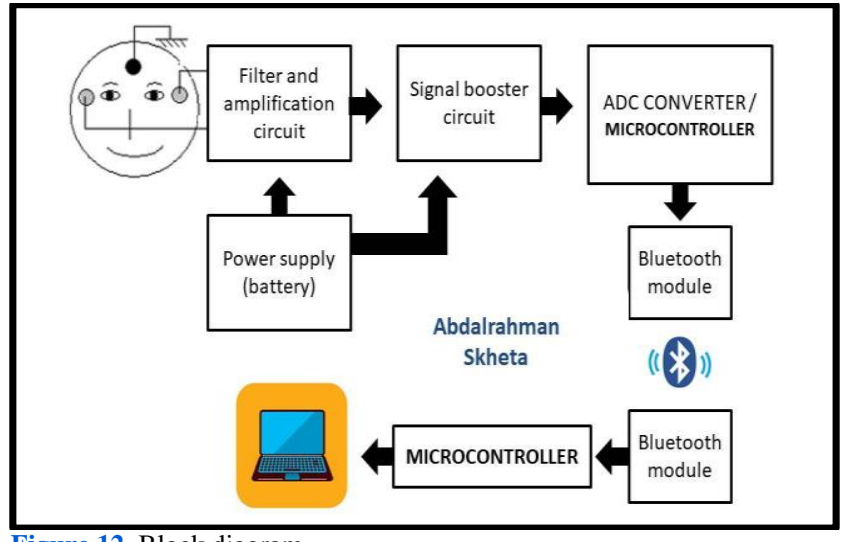

Figure 12. Block diagram

\subsubsection{Electrodes}

AG/AG-CL electrodes were used. The AG/ AG-CL electrode is one of the most widely used electrodes to obtain the EOG signal. Figure 13 shows one of the electrodes that were used to obtain the signal. Where silver used must be $99.99 \%$ pure.

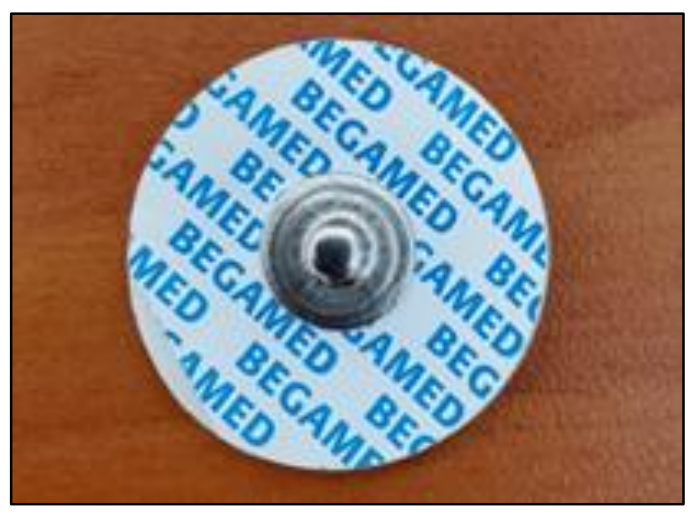

Figure 13. AG / AG-CL electrode

\subsubsection{Connecting cables}

To reduce noise ECG device cable was used. Figure 14 shows the used cable

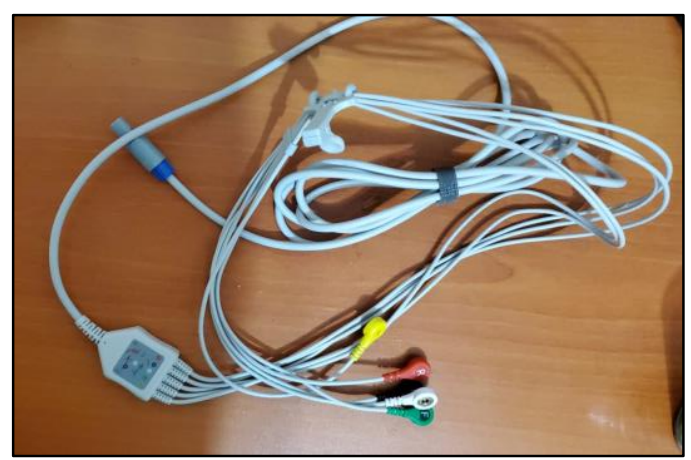

Figure 14. ECG cable

\subsubsection{PCB}

A PCB for the main circuit has been designed. The circuit is made of two layers with $7.5 \times 8.5 \mathrm{~cm}$ dimensions. The circuit consists of three main sections: horizontal EOG signal processing section, vertical EOG signal processing section, and third section.

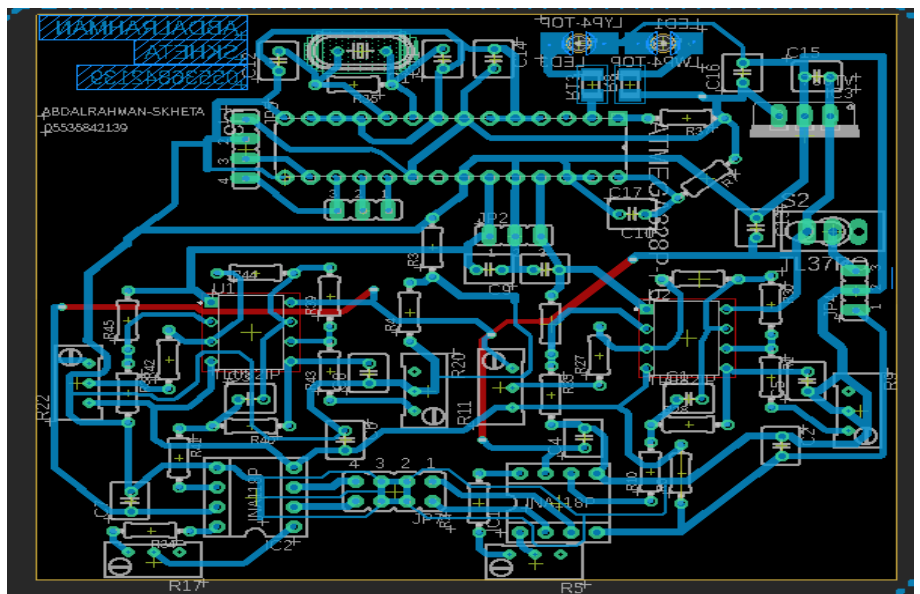

Figure 15. The board after drawing it

The third section contains the AT-MEGA328 microprocessor and HC-05 Bluetooth module. It also has a voltage regulator to feed the processor and Bluetooth module at a voltage of $5 \mathrm{~V}$. Horizontal \& vertical EOG signal processing sections are identical, both contain INA118 amplifier and TL082 amplifier, in addition to passive components such as capacitors and resistors. The circuit is drawn using EAGLE program [27]. Figure 15 shows PCB after drawing it. Figure 16 shows front and back of PCB after it has been printed.

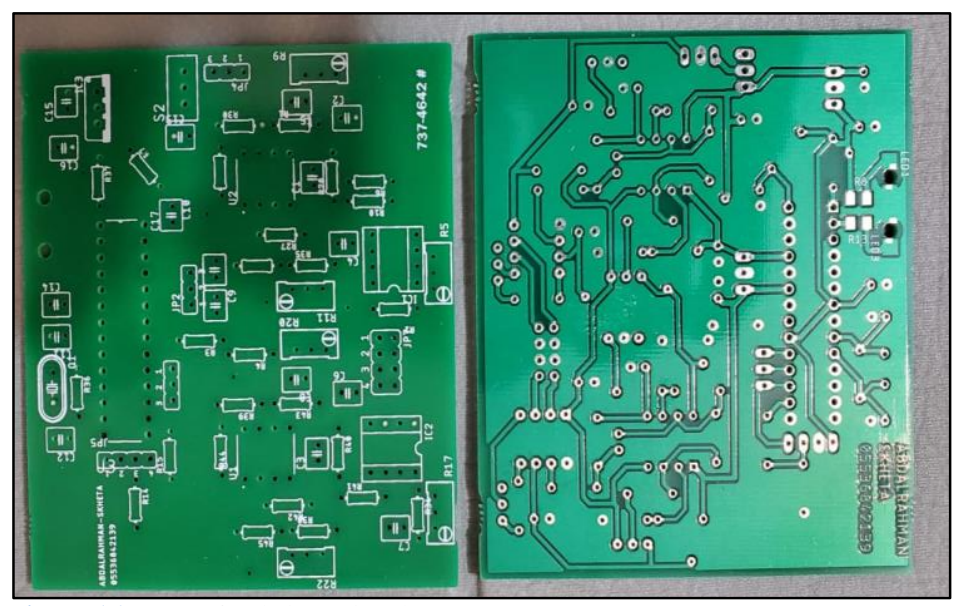

Figure 16. PCB, after being printed

\subsection{Code flowchart}

The flowchart shows how code works. After obtaining horizontal and vertical analog signals, they are converted into a digital signal and stored in two variables: (Xreading) for vertical signal and (Yreading) for horizontal signal. Looking ahead saved as a center point and stored in a variable called (center). To ensure that there is no interference (interfering signals) such as eyelid movement and noise signals from surrounding muscles, a constant so-called (threshold) was used. Threshold subtracts from the value of the obtained signal. 


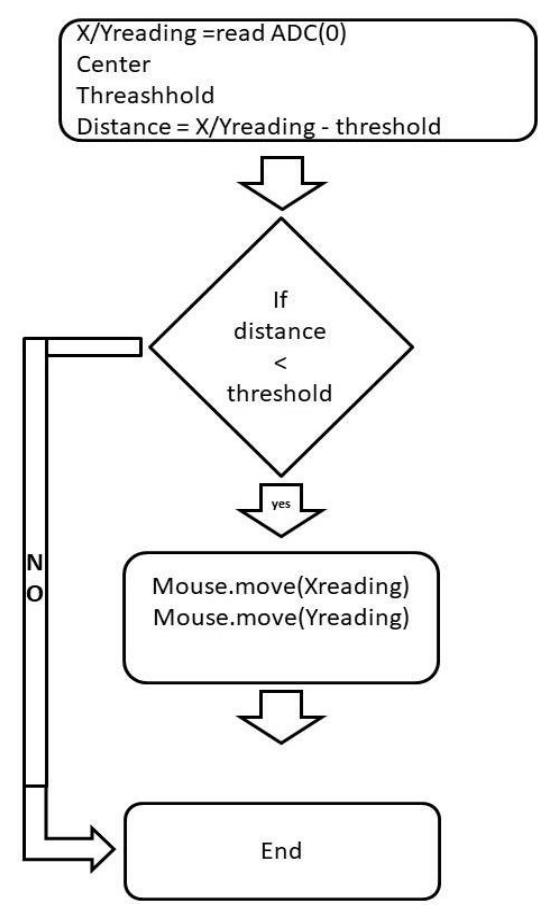

Figure 17. Code flowchart

The microcontroller determines the location of the eye according to the final value, then gives orders to move the mouse cursor accordingly. Figure 17 shows the Code flowchart.

\section{RESULTS}

After electronic elements were placed on the circuit, electrodes were attached to the eye area and fed to the circuit, then signals obtained at each stage were examined (after amplification, after filtering, and after raising signal value). As explained previously, the circuit contains three main sections. Two of them are similar: the horizontal EOG acquisition circuit and the vertical EOG acquisition circuit. As results will be the same in the second section, EOG signal in the first section was studied. Since the circuit is complex and contains many elements, the first section in a simplified way was drawn, where passive components such as resistor and capacitor were removed.

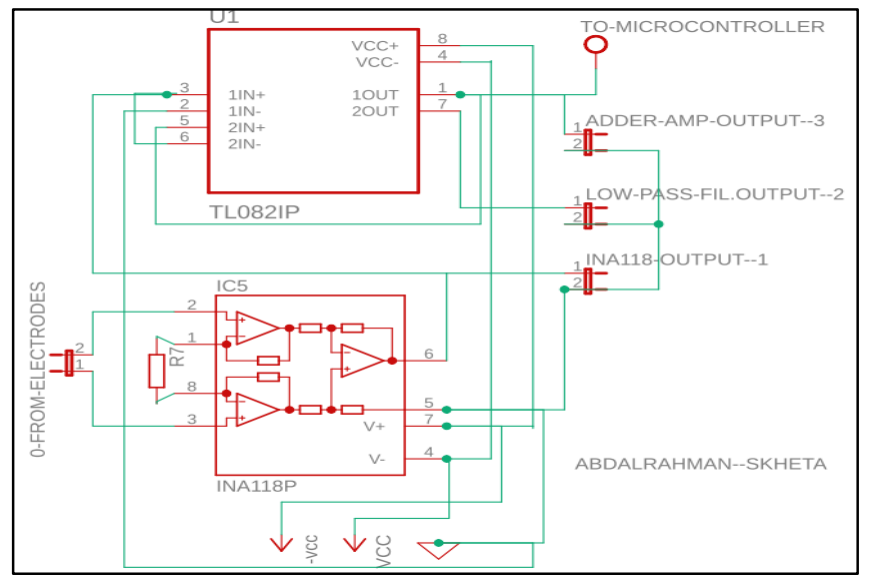

Figure 18. Simple diagram of the signal acquisition section

Figure 18 shows a simplified diagram of the signal acquisition section. Number 0 jumper is the input of INA118 [28] (where electrodes are connected). Number 1 jumper shows the output of INA118. Number 2 jumper shows the output of TL082 [29] (low pass filter) where the output of this amplifier will be the input of adder amplifier. Number 3 jumper shows the output of the adder amplifier and it is the final signal that will be converted into a digital signal, so it is connected to the microcontroller.

The oscilloscope probe was connected to jumper number 1 and the person who uses the device began to look left and right. The result is observed to be good, but the noise is still high. The obtained signal is shown in Figure 19.

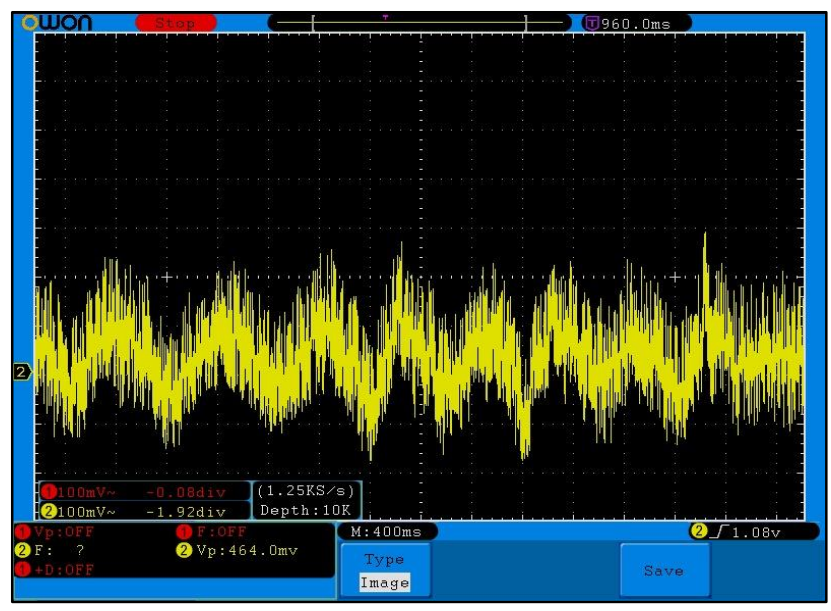

Figure 19. INA118 output

Afterward, the oscilloscope probe was connected to jumper number 2 (low pass filter output) and the person who uses the device started to look left and right. In this case, the noise level was reduced by $95 \%$, the signal was low noise and clear. Figure 20 shows the state of the signal after filtering.

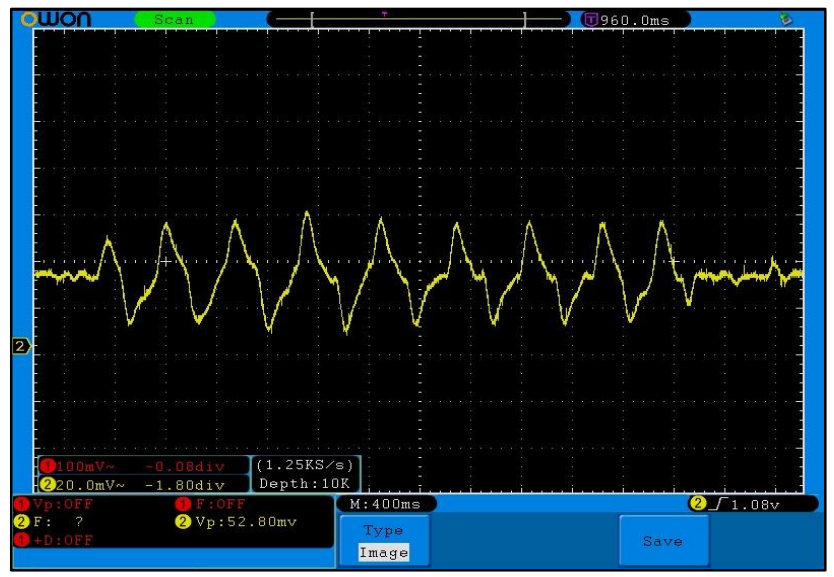

Figure 20. The output after it has been filtered

Then the person looked to the far-right for a second, and then looked ahead. A positive pulse with low noise is displayed on the oscilloscope screen. Figure 21 shows a positive pulse. 


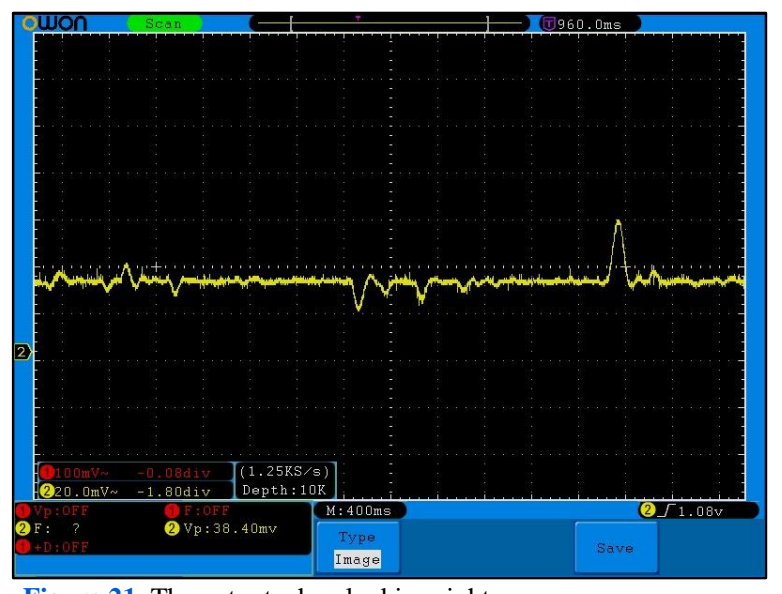

Figure 21. The output when looking right

Next, the person wearing the electrode looked far left for one second, then forward. A low-noise negative pulse is displayed on the oscilloscope screen. Figure 22 shows a negative pulse. In this way, a clear signal that can be used was obtained. Then the negative side of the signal was raised to a positive value by the adder amplifier. After increasing the value of the signal, it was converted into a digital signal.

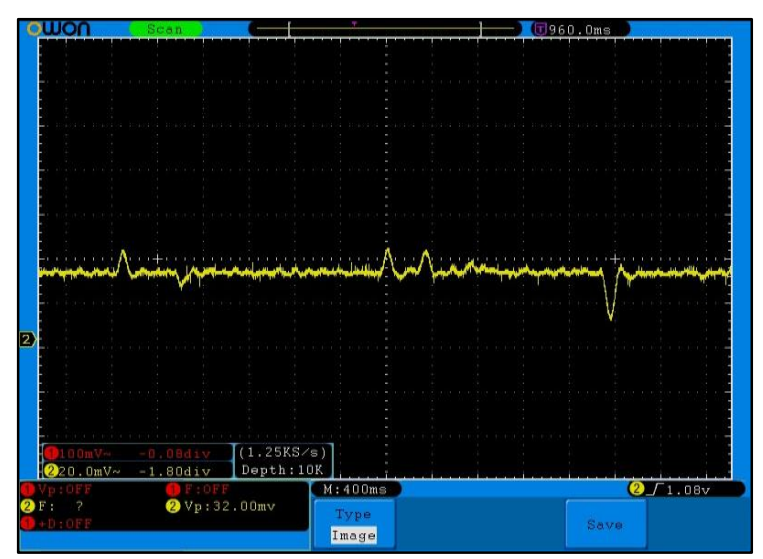

Figure 22. The output when looking left

The EOG signal value must be positive. The second amplifier in TL082 circuit was used to raise the negative portion of the signal from negative to positive. The value of primitive potential (Electric potential when looking forward) was calibrated at $2.5 \mathrm{~V}$ by changing the value of variable resistance that connected to the amplifier. Figure 23 shows the output of the adder amplifier when looking forward.

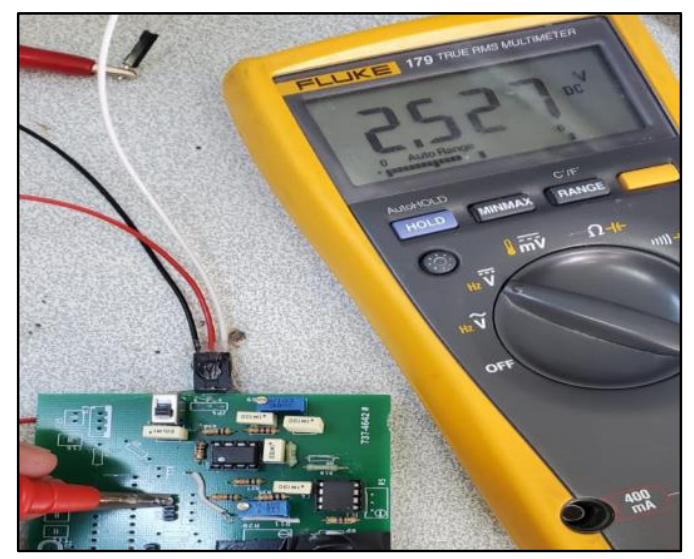

Figure 23. Adjust output at $2.5 \mathrm{~V}$ when looking straight
TABLE II

The OUTPut Changes ACCORding to Eye MOVEMENT

\begin{tabular}{ll}
\hline \hline Eye direction & Output(Volt) \\
\hline Forward & 2.5 \\
Right (25 degrees) & 2.7 \\
Far-Right & 2.9 \\
Left (25 degrees) & 2.3 \\
Far-Left & 2.1 \\
& \\
\hline \hline
\end{tabular}

The final results show that the circuit output is positive in all cases. This means that the resulting EOG signal can be processed by the processor.

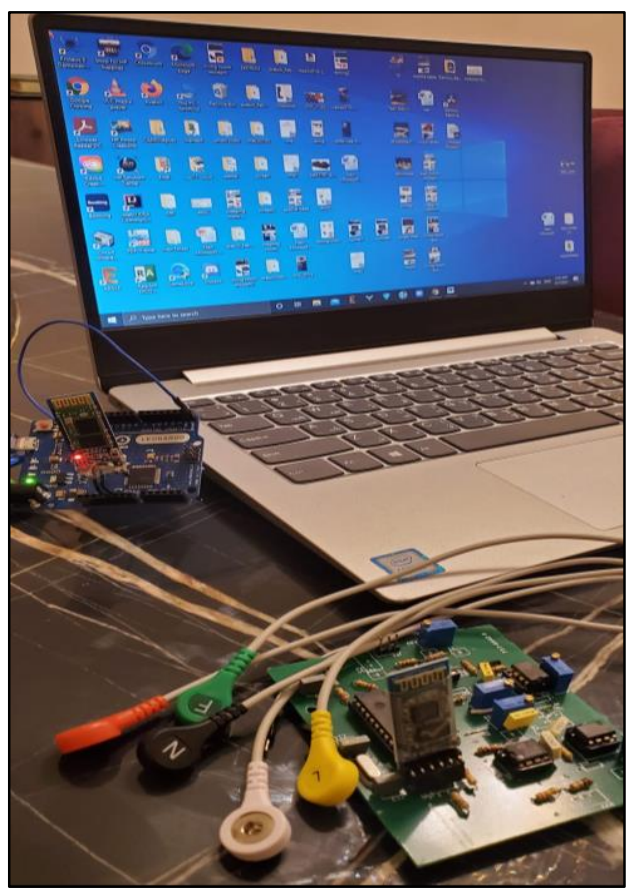

Figure 24. The final shape of the system

Since the output value is in the range of (0-5) volts, the processor can convert this signal to a digital signal. In this way, a positive and pure EOG signal is obtained. The final signal is effective and usable. Figure 24 shows final shape of the system when connected to a computer. After calibrating the adder amplifier at $2.5 \mathrm{~V}$ when looking forward, the output voltage was measured when looking right with a small degree, far right, left with a small degree, and far left. The circuit output is shown in Table 2.

\section{CONCLUSION}

Medical assistance systems can be used in many cases and diseases such as visual impairment, mobility disability, paralysis, deafness. Creating an assistive system using vital signals for people with special needs can provide many benefits and advantages. In recent years, the use and dependence of vital signals have increased due to their effectiveness and easy acquisition. The designed system has many advantages over systems designed for the same purpose. The main advantages of the system:

- The system is wireless, this provides more convenience to the user. Since there is no cable connecting the patient to the computer, the patient can use the computer more comfortably. 
- Two processors are used in the system. The first processor is for processing the EOG signal, the second processor is for controlling the computer. Using two processors ensures the speed of the system. In this way, the user can control the computer smoothly and effectively.

Several steps were taken to obtain an effective signal:

- A special PCB is designed to separately process the horizontal and vertical EOG signal.

- The circuit is powered by a DC current to reduce noise.

- Special cables were used to effectively acquire the EOG signal and reduce signal loss during transmission from the patient's body to the electrical circuit.

- The negative part of the EOG signal was shifted to a positive value and the signal was filtered with active filters.

\section{ACKNOWLEDGEMENT}

This study has been produced from the Master's degree dissertation of Abdalrahman SKHETA.

\section{REFERENCES}

[1] M.Merino \& O. Rivera\& I. Gómez \&A. Molina. A Method of EOG Signal Processing to Detect the Direction of Eye Movements. First international conference on sensor device .2010.

[2] A. Naga Rajesh, S. Chandralingam, T. Anjaneyulu, K. Satyanarayana. EOG Controlled Motorized Wheelchair for Disabled Persons, vol. 115 ,pp. 965-970, 2014.

[3] B.Narayanan. Micro controller based EOG guided wheelchair-January 33(2), 3398-3401, 2013.

[4] N. ÖZKAN , A.IŞIK , U. FIDAN. Design and Acquisition of EOG Based Interactive Communications for ALS Patients, Electronics And Computers Special Issue, 2017.

[5] L. Y., Hsu, C.-L., Lin, T.-C., Tuan, J.-S., \& Chang, S.-M. EOG-based Human-Computer Interface system development. Expert Systems with Applications37(4), 3337-3343, 2010.

[6] Barea, R., Boquete, L., Ortega, S., López, E., \& Rodríguez-Ascariz, J. M. (2012). EOG-based eye movements codification for human computer interaction, 39(12), pp. 2677-2683, 2012.

[7] Postelnicu, C.-C., Girbacia, F., \& Talaba, D. (2012). EOG-based visual navigation interface development. 39(3), 2677-2683.

[8] Schlögl, A., Keinrath, C., Zimmermann, D., Scherer, R., Leeb, R., \& Pfurtscheller, G. (2007). A fully automated correction method of EOG artifacts in EEG recordings. DOI:10.1016/j.clinph.2006.09.003.

[9] RAMIS İLERI: Determination of the most appropriate writing characters for the education of children with dyslexia by using EOG signals- UlusalTezMerkezi - 2020.

[10] AHMET COŞKUN:Sleep staging with electrooculography (EOG) signals, 22-35, 2017.

[11] Eye anatomy, parts of the eye and how we see, 2021. https://www.aao.org/eye-health/anatomy/parts-of-eye

[12] Eye anatomy: close look at the parts of the eye. 2021. https://www.allaboutvision.com/resources/anatomy.htm

[13] M.C Kiernan, S. Vucic, B. C. Cheah, M. R. Turner, A., Orla, J. R Burrell, M.C Zoing. Amyotrophic lateral sclerosis 7, 2011. DOI:10.1016.

[14] Berthoz A, Melvill Jones G Büttner.Ennever JA. The Electric Signals Originating in the Eye.

[15] Estrany, B., Fuster, P., Garcia, A., \& Luo, Y. (2009). EOG signal processing and analysis for controlling computer by eye movements. DOI:10.1145/1579114.1579132.

[16] Abo-Zahhad, M., Ahmed, S. M., \& Abbas, S. N. (2015). A New EEG Acquisition Protocol for Biometric Identification Using Eye Blinking Signals, 2015.06.05.

[17] Mala Sundaram Feature selection in activity recognition using eye movements: Filter based approach Jan 2015.

[18] Ma, J., Zhang, Y., Cichocki, A., \& Matsuno, F. (2015). A Novel EOG/EEG Hybrid Human-Machine Interface Adopting Eye Movements and ERPs: Application to Robot Control. tbme.2014.2369483.

[19] Ivailo Milanov Pandiev-Analysis and Design of Instrumentation Amplifiers-June 2013. DOI: 10.1125

[20] T. Dear, J. Chang, W. Chow, D. Wai, J. Wang-Filters -Summer 2012.
[21] A. Goen.Analysis and Design of Low Pass Filter by Using DGS for WLAN Application-January 2007 DOI:10.15662.

[22] W. Hendra Gunawan STT Migas. Low Pass Filter Design With Artificial Ground Structure-November 2017:10.32487.

[23] R. Reddy R. PERFORMANCE ANALYSIS OF ADDER CIRCUITS USING FINFET'S. January 2017 DOI:10.26634.

[24] James M. Fiore. Operational Amplifiers \& Linear Integrated Circuits: Theory and Application-Version 3.2.5, 15 December 2020.

[25] ATmega328P 8-bit AVR Microcontroller with 32K Bytes In-System Programmable Flash. https://datasheetspdf.com/datasheet/ATmega328P.html

[26] HC-05 -Bluetooth to Serial Port Module. http://www.electronica60norte.com/mwfls/pdf/newBluetooth.pdf

[27] autodesk.com/products/eagle/overview.

[28] INA118 Precision, Low-Power Instrumentation Amplifier-Datasheet production data. https://www.ti.com/lit/ds/symlink/ina118.pdf?ts=1636968472131\&ref url=https\%253A\%252F\%252Fwww.google.ru\%252F

[29] t1082- General purpose JFET dual operation amplifiers -Datasheet production data. https://www.ti.com/lit/ds/symlink/t1082-n.pdf

\section{BIOGRAPHIES}

Abdalrahman SKHETA received his bachelor's degree in medical electronics engineering from Aleppo University, Syria in 2017. After obtaining a C1 certificate in Turkish, he began studying for a master's degree at Marmara University in 2019. In 2020 Participated in the 9th International Conference on Advanced Technologies With an article entitled: Study and Design of Power Supply Based on Pettier to Charge the EV Auxiliary Battery. The article was published in the IJEAT journal.

Nazmi EKREN received the B.S. degree from the Electrical Engineering Department, Yildiz Technical University (YTU), Istanbul, Turkey, in 1987, and the M.S. degrees from the Marmara University in 1989 and $\mathrm{PhD}$ degrees from the Istanbul University in 1996. He is currently an associate professor at the Marmara University. His research interests PV systems, fabrication at Nano level and lighting. He works on tissue engineering applications, 3Dbioprinting, electrospinning. 